\title{
Turfgrass Monoculture, Cool-Cool, and Cool-Warm Season Seed Mixture Establishment and Growth Responses
}

\author{
Hassan Salehi ${ }^{1}$ and Morteza Khosh-Khui \\ Department of Horticultural Science, College of Agriculture, Shiraz University, \\ Shiraz, Iran
}

Additional index words. Cynodon, Festuca, Lolium, Poa, fall sowing, spring sowing, species

\begin{abstract}
Turfgrass seeds can be sown individually, in mixes, or overseeded to provide green color and uniform surfaces in all the seasons. This investigation was conducted to compare different turfgrass species and their seed mixtures. In this research, the turfgrasses-perennial ryegrass (Lolium perenne $\mathbf{L}$. 'Barball'), kentucky bluegrass (Poa pratensis $\mathbf{L}$. 'Merion'), common bermudagrass (Cynodon dactylon [L.] Pers.), and strong creeping red fescue (Festuca rubra L. var. rubra 'Shadow')—in monoculture or in mixtures of 1:1 (by weight) and a 1:1:1:1 (by weight) and two sport turfgrasses-BAR 11 (Barenbrug Co.) and MM (Mommersteeg Co.)-were used. The seeds were sown in March and October (spring and fall sowing) in 1998 and 1999. The experiments were conducted in a split-split block design with year as main plot, sowing season as subplot, and turfgrass types as subsubplot. The turfgrasses were compared by measuring visual quality, chlorophyll index after winter and summer, rooting depth, verdure and/or root fresh and dry weight, tiller density, and clippings fresh and dry weight. Fall sowing was superior to spring sowing and resulted in greater root growth, clipping yield, and chlorophyll content. Poa+Cynodon seed mixture was the best treatment and had high tiller density, root growth, and chlorophyll content. Lolium and Festuca monocultures, and Poa+Festuca and Cynodon+Festuca seed mixtures were not suitable with regard to low tiller density, sensitivity to high temperatures, low root growth, and low tiller density, respectively. The cool-warm-season seed mixture (Poa+Cynodon) can be used alternatively in overseeding programs in the areas with soil and environmental conditions similar to this research site.
\end{abstract}

Lawn and turfgrasses are important for recreational and ornamental uses (Beard, 1973). There are several reports on the comparison and selection between different genotypes of turfgrasses for color, uniformity, wear tolerance, and coverage (Dunn et al., 1994; Gooding and Newell, 1991; Misiha, 1991; Newell et al., 1996; Skirde, 1989). Misiha (1991) compared different turfgrass seed mixtures and showed that the mixture of Poa pratensis L. 'Entopper' with Festuca rubra L. 'Hareld' had the best establishment rate and highest chlorophyll content, plant density, and cut dry weight. Newell et al. (1996) indicated that Lolium and Festuca seed mixtures had the best wear tolerance. Comparisons were also made between new cultivars of Festuca arundinacea Schreb., in monoculture or in mixtures with Poa pratensis L. and Lolium perenne L. cultivars where Festuca was found to have poor competitiveness against Lolium and Poa (Skirde, 1989).

Hybrid bermudagrass greens are normally overseeded with ryegrass in the fall to provide green color, uniform surfaces and wear resis-

Received for publication 25 Aug. 2003. Accepted for publication 17 Mar. 2004. The authors wish to thank H. Azarakhsh, M. Fadaie (deceased), M.H. Safarzadeh, F. Nikbakht, A. Shahsavari and P. Roashantabari for technical assistance and Z. Seddighi for typing the manuscript. Research Council of Shiraz University provided support for this project (no.: 76-AG-1008-599).

${ }^{1}$ To whom reprint requests should be addressed; e-mail hsalehi@shirazu.ac.ir. tance during the period of winter dormancy (Mazur, 1988). Availability of newer, more competitive cool-season grasses have renewed interest in persistent, balanced warm- and cool-season species mixtures for sports turf (Dunn et al., 1994). Few reports are available on replacing these time-consuming overseeding programs with alternative methods. Dunn et al. (1994) recommended seed mixtures of cool-season turfgrasses with Cynodon dactylon [L.] Pers. for the best wear tolerance. The objectives of this research were to compare monoculture, cool-cool-season and cool-warm-season turfgrass seed mixtures with regard to visual, morphological, and physiological characters.

\section{Materials and Methods}

Investigations were conducted at the Experimental Farm of the Department of Horticultural Science, College of Agriculture, Shiraz University, Shiraz, Iran, at Bajgah, $1810 \mathrm{~m}$ above the mean sea level, $52^{\circ} 32^{\prime} \mathrm{E}$ and $29^{\circ} 36^{\prime} \mathrm{N}$, with Daneshkadeh soil series (fine, mixed, mesic, Calcixerollic Xerochrepts, $\mathrm{pH}$ $=8$ ), from 1998 to 2000. The meteorological data of the experimental site is shown in Table 1. Long-term average of maximum and minimum temperatures are $38^{\circ} \mathrm{C}$ and $-9^{\circ} \mathrm{C}$, respectively and yearly precipitation at this site is $400 \mathrm{~mm}$.

The experiments were conducted in a $2 \times 2$ $\times 13$ split-split block factorial design with year (1998 and 1999) as main plot, sowing season (spring and fall) as subplot and turfgrass types as sub-subplot, and four replications in each treatment. Individual plots measured $4 \mathrm{~m}^{2}$ (1 $\times 4 \mathrm{~m}$ ). Turfgrasses were perennial ryegrass (Lolium perenne L. 'Barball'), kentucky bluegrass (Poa pratensis L. 'Merion'), common bermudagrass [Cynodon dactylon [L.] Pers. (California origin)], and strong creeping red fescue (Festuca rubra L. var. rubra 'Shadow') in monoculture or in mixtures of 1:1 (by weight) or $1: 1: 1: 1$ (by weight) and two sport turfgrasses-BAR 11 [consisting 40\% perennial ryegrass, Lolium perenne L. 'Barmona', $40 \%$ strong creeping red fescue, Festuca rubra L. var. rubra 'Rumba' and $20 \%$ chewing fescue, Festuca rubra L. var. commutata 'Baruba' (Barenbrug Co.)] and MM [consisting 20\% kentucky bluegrass 'Geronimo', 20\% slender creeping red fescue (Festuca rubra L. var. trichophylla 'Scorpio'), 30\% strong creeping red fescue 'Commodore', 25\% perennial ryegrass 'Rival', and 5\% colonical bentgrass (Agrostis tenuis Sibth 'Highland Bent') (Mommersteeg Co.). Thus, turfgrass treatments were abbreviated as Lolium $=\mathrm{L}, P o a=\mathrm{P}$, Cynodon $=\mathrm{C}$, Festuca = F, BAR 11, MM, L+P, L+C, $\mathrm{L}+\mathrm{F}, \mathrm{P}+\mathrm{C}, \mathrm{P}+\mathrm{F}, \mathrm{C}+\mathrm{F}$, and $\mathrm{L}+\mathrm{P}+\mathrm{C}+\mathrm{F}$.

Turfgrass plots were established by directly sowing the seeds at two times, March and October, in both 1998 and 1999 years. The rate of seeding was $50 \mathrm{~g} \cdot \mathrm{m}^{-2}$ for $\mathrm{L}$ and $\mathrm{F}, 40 \mathrm{~g} \cdot \mathrm{m}^{-2}$ for BAR 11 and $\mathrm{MM}, 30 \mathrm{~g} \cdot \mathrm{m}^{-2}$ for $\mathrm{P}$ and $\mathrm{C}, 20+20$ $\mathrm{g} \cdot \mathrm{m}^{-2}$ for $\mathrm{L}+\mathrm{P}, \mathrm{L}+\mathrm{C}, \mathrm{L}+\mathrm{F}, \mathrm{P}+\mathrm{C}, \mathrm{P}+\mathrm{F}$, and $\mathrm{C}+\mathrm{F}$ and $10+10+10+10 \mathrm{~g} \cdot \mathrm{m}^{-2}$ for $\mathrm{L}+\mathrm{P}+\mathrm{C}+\mathrm{F}$. The plots were prepared after plowing and leveling the soil. The plots were hand-seeded and immediately covered with a thin layer (about 0.5 $\mathrm{cm}$ ) of decomposed horse manure. Irrigation was applied regularly during establishment and then only during soil surface drought periods thereafter. During the experiment period, all plants were clipped when needed, from $3 \mathrm{~cm}$ above the ground by a hand-mower. The grass clippings were not returned to the plots.

Measurements. Visual quality (Beard, 1973) was assessed periodically throughout and after each growing season using a ranking scale of 1 to $11,1=$ no live turf; $11=$ ideal shoot density, winter and summer color, and uniformity.

Tiller density, verdure and/or root fresh and dry weight and clippings fresh and dry weight at August, each year and chlorophyll index (Beard, 1973) after winter and summer (March and September, respectively) and mean rooting depth (average length of roots), all were measured in $100-\mathrm{cm}^{2}$ subsamples in each plot. For measuring the dry weight, the materials were dried at $60^{\circ} \mathrm{C}$ for $48 \mathrm{~h}$. Random subsample of each plot was collected using a $10 \times 10 \times 10$-cm metal block inserted into the soil. Then, the samples were soaked in tap water and soil was removed. Cleaned and ambient-air dried samples of plants were transferred to the laboratory for further measurements. Clippings and verdures were collected $20 \mathrm{~d}$ after previous mowing from $3 \mathrm{~cm}$ above the ground, by a hand-mower. Chlorophyll index was measured by spectrophotometric method (Horwitz, 1975) at 645 and $663 \mathrm{~nm}$ wavelengths.

Statistical analysis. All data (except visual 
Table 1. Monthly average precipitation and temperatures at the experimental site for April 1998 to March 2000.

\begin{tabular}{|c|c|c|c|c|c|c|}
\hline \multirow[b]{2}{*}{ Month } & \multicolumn{2}{|c|}{1998} & \multicolumn{2}{|c|}{1999} & \multicolumn{2}{|c|}{2000} \\
\hline & $\begin{array}{l}\text { Precipitation } \\
(\mathrm{mm})\end{array}$ & $\begin{array}{l}\text { Temp } \\
\left({ }^{\circ} \mathrm{C}\right)\end{array}$ & $\begin{array}{l}\text { Precipitation } \\
(\mathrm{mm})\end{array}$ & $\begin{array}{l}\text { Temp } \\
\left({ }^{\circ} \mathrm{C}\right)\end{array}$ & $\begin{array}{l}\text { Precipitation } \\
\quad(\mathrm{mm})\end{array}$ & $\begin{array}{c}\text { Temp } \\
\left({ }^{\circ} \mathrm{C}\right)\end{array}$ \\
\hline April & 55.0 & 12.0 & 10.0 & 12.8 & 7.0 & 12.7 \\
\hline May & 5.0 & 16.2 & 0.0 & 17.3 & 0.0 & 17.5 \\
\hline June & 0.0 & 20.5 & 0.0 & 21.0 & 0.0 & 19.7 \\
\hline July & 0.0 & 24.6 & 7.0 & 23.3 & 0.0 & 23.2 \\
\hline August & 0.0 & 23.7 & 0.0 & 23.5 & 0.0 & 22.3 \\
\hline September & 0.0 & 20.8 & 0.0 & 21.1 & 0.0 & 20.2 \\
\hline October & 0.0 & 16.7 & 0.0 & 15.3 & 8.0 & 15.3 \\
\hline November & 0.0 & 10.7 & 0.0 & 11.3 & 67.0 & 10.2 \\
\hline December & 0.0 & 7.5 & 24.0 & 6.8 & 104.0 & 6.0 \\
\hline January & 118.0 & 6.5 & 111.0 & 4.1 & 39.0 & 4.2 \\
\hline February & 113.0 & 5.3 & 73.0 & 4.1 & 39.0 & 3.5 \\
\hline March & 190.0 & 7.0 & 0.0 & 7.0 & 15.0 & 8.0 \\
\hline
\end{tabular}

Table 2. Visual quality of spring and fall sown turfgrass types averaged over 2 years of growing in this study.

\begin{tabular}{lcc}
\hline \multirow{2}{*}{$\begin{array}{l}\text { Turfgrass } \\
\text { type }\end{array}$} & \multicolumn{2}{c}{ Visual quality } \\
\cline { 2 - 3 } & Spring sowing & Fall sowing \\
\hline L & $5.0 \mathrm{f}^{\mathrm{x}}$ & $7.8 \mathrm{~d}$ \\
$\mathrm{P}$ & $6.0 \mathrm{~d}$ & $6.7 \mathrm{~g}$ \\
$\mathrm{C}$ & $6.0 \mathrm{~d}$ & $8.6 \mathrm{~b}$ \\
$\mathrm{~F}$ & $3.0 \mathrm{~h}$ & $5.0 \mathrm{j}$ \\
BAR 11 & $6.8 \mathrm{~b}$ & $6.0 \mathrm{~h}$ \\
MM & $5.1 \mathrm{f}$ & $7.7 \mathrm{e}$ \\
$\mathrm{L}+\mathrm{P}$ & $7.4 \mathrm{a}$ & $9.2 \mathrm{a}$ \\
$\mathrm{L}+\mathrm{C}$ & $5.4 \mathrm{e}$ & $8.0 \mathrm{c}$ \\
$\mathrm{L}+\mathrm{F}$ & $6.5 \mathrm{c}$ & $7.8 \mathrm{de}$ \\
$\mathrm{P}+\mathrm{C}$ & $7.5 \mathrm{a}$ & $9.2 \mathrm{a}$ \\
$\mathrm{P}+\mathrm{F}$ & $3.1 \mathrm{~h}$ & $5.2 \mathrm{i}$ \\
$\mathrm{C}+\mathrm{F}$ & $3.4 \mathrm{~g}$ & $5.2 \mathrm{i}$ \\
$\mathrm{L}+\mathrm{P}+\mathrm{C}+\mathrm{F}$ & $6.8 \mathrm{~b}$ & $7.5 \mathrm{f}$ \\
Mean & 5.5 & $7.2^{* *}$ \\
\hline
\end{tabular}

${ }^{\mathrm{z}} 11$ = Ideal shoot density, winter and summer color, and uniformity; $1=$ no live turf.

${ }^{\mathrm{y}} \mathrm{L}=$ Lolium, $\mathrm{P}=$ Poa, $\mathrm{C}=$ Cynodon, $\mathrm{F}=$ Festuca, BAR 11 and $\mathrm{MM}=$ sport turfgrasses.

'In each column, means followed by the same letter are not significantly different according to Duncan's multiple range test at $1 \%$ level.

${ }^{* *}$ Statistically different at $P \leq 0.01$.

quality data) were subjected to an analysis of variance (ANOVA) using MSTATC software program with treatment sum of squares partitioned into year, sowing season, turfgrass type, and interaction effects among them. One-way ANOVA of the same software was used for visual quality data. Duncan's multiple range test at $5 \%$ and $1 \%$ levels was used for mean separation.

\section{Results}

Visual quality. Observations indicate the superiority of fall sowing than spring sowing (Table 2). The $\mathrm{L}+\mathrm{P}$ and $\mathrm{P}+\mathrm{C}$ had the highest and $\mathrm{F}, \mathrm{P}+\mathrm{F}$, and $\mathrm{C}+\mathrm{F}$ had the lowest quality, in both sowing times.

Sowing time. Generally, fall sowing was superior to spring sowing (Table 3 ). There were no significant differences between two sowing times with regard to tiller density, and verdure fresh and dry weight (Table 3 ).

Turfgrass type. Comparison between different turfgrass types due to measured variables is shown in Tables 4 and 5 (means are averaged over the two sowing times). Among the turfgrass type, there were no significant depth, and chlorophyll index after summer.

- $\mathrm{C}+\mathrm{F}$ : This turfgrass had high total and root dry weight, and rooting depth but had low tiller density and was weak according to other measured variables.

- $\mathrm{L}+\mathrm{P}+\mathrm{C}+\mathrm{F}$ : This type was high growing with regard to several measured variables except the low tiller density and total and root fresh weight.

Interaction between sowing time and turfgrass type. The interaction between sowing times and turfgrass types with regard to the measured variables are shown in Tables 6 and 7. Results indicated that interaction of two sowing times and turfgrass types had significant effects on the other variables. Festuca has the highest tiller density in both sowing times, but has no significant difference with $\mathrm{P}, \mathrm{C}$, and $\mathrm{P}+\mathrm{C}$. Clipping fresh weight of all turfgrass types were greater in fall sowing than spring sowing cultures. Generally, mean rooting depth was greater in fall sowing than spring sowing cultures. Most turfgrass types had greater chlorophyll content after summer and winter in fall culture than spring culture. Festuca has the lowest chlorophyll index after winter in spring culture. Cynodon has higher chlorophyll index after winter in fall culture compared to spring culture.

\section{Discussion} chlorophyll inex after summer (Table 5).

- BAR 11 and MM: These sport turfgrasses had low tiller density. Generally, shoot and root growth was greater in BAR 11 compared to MM.

- L+P: This turfgrass type had high rooting depth but was low in tiller density, total fresh weight, root fresh and dry weight, and clipping dry weight.

- $\mathrm{L}+\mathrm{C}$ : As above $(\mathrm{L}+\mathrm{P})$ in addition to having low clipping fresh weight.

- L+F: This type had high verdure fresh and dry weight, clipping fresh and dry weight, and rooting depth but was low in tiller density, and total and root fresh and dry weight.

- P+C: This turfgrass was fast growing with regard to several measured variables except the low clipping dry weight.

- P+F: This type had low verdure fresh and dry weight, total fresh weight, rooting
In this study, visual quality measurements indicated the superiority of fall sowing over spring sowing. Visual quality may not be enough without attention to the other measurements because visual rating systems are subjective and consequently the results they produce can be inconsistent (Horst et al., 1984). Although, Beard (1973) is convinced that visual qualifying is the best procedure for selection between turfgrasses. Garling and Boehm (2001) used only turfgrass color for visual quality measurements and Cooper and Spokas (1991) stated that color is the most important characteristics that contribute to visual turfgrass quality. In present investigation, results of visual quality studies were confirmed by other measurements such as higher shoot, root, and clipping yield in fall sowing compared to spring sowing.

Table 3. Comparisons between two sowing times, according to measured variables in different turfgrass types in $100 \mathrm{~cm}^{2}$ area averaged over 2 years of growing in this study.

\begin{tabular}{lrr}
\hline & \multicolumn{2}{c}{ Sowing time } \\
\cline { 2 - 3 } Variable & \multicolumn{1}{c}{ Spring } & Fall \\
\hline Tiller density $\left(\right.$ number $\left./ 100 \mathrm{~cm}^{2}\right)$ & $463.1 \mathrm{a}^{\mathrm{z}}$ & $474.1 \mathrm{a}$ \\
Total fresh weight $(\mathrm{shoot}+\mathrm{root})\left(\mathrm{g} / 100 \mathrm{~cm}^{2}\right)$ & $27.5 \mathrm{~B}$ & $38.9 \mathrm{~A}$ \\
Total dry weight $(\mathrm{shoot}+\mathrm{root})\left(\mathrm{g} / 100 \mathrm{~cm}^{2}\right)$ & $9.6 \mathrm{~B}$ & $13.6 \mathrm{~A}$ \\
Verdure fresh weight $\left(\mathrm{g} / 100 \mathrm{~cm}^{2}\right)$ & $14.1 \mathrm{a}$ & $15.4 \mathrm{a}$ \\
Verdure dry weight $\left(\mathrm{g} / 100 \mathrm{~cm}^{2}\right)$ & $4.4 \mathrm{a}$ & $4.7 \mathrm{a}$ \\
Root fresh weight $\left(\mathrm{g} / 100 \mathrm{~cm}^{2}\right)$ & $13.5 \mathrm{~B}$ & $23.5 \mathrm{~A}$ \\
Root dry weight $\left(\mathrm{g} / 100 \mathrm{~cm}^{2}\right)$ & $5.3 \mathrm{~B}$ & $8.9 \mathrm{~A}$ \\
Clipping fresh weight $\left(\mathrm{g} / 100 \mathrm{~cm}^{2}\right)$ & $0.5 \mathrm{~B}$ & $0.8 \mathrm{~A}$ \\
Clipping dry weight $\left(\mathrm{g} / 100 \mathrm{~cm}^{2}\right)$ & $0.1 \mathrm{~B}$ & $0.2 \mathrm{~A}$ \\
Mean rooting depth $(\mathrm{cm})$ & $6.0 \mathrm{~b}$ & $7.1 \mathrm{a}$ \\
Chlorophyll index & & \\
$\quad$ After summer $\left(\mathrm{mg} / 100 \mathrm{~cm}^{2}\right)$ & $22.6 \mathrm{a}$ & $27.8 \mathrm{a}$ \\
$\quad$ After winter $\left(\mathrm{mg} / 100 \mathrm{~cm}^{2}\right)$ & $5.1 \mathrm{~B}$ & $7.9 \mathrm{~A}$ \\
\hline
\end{tabular}

${ }^{2}$ In each row, means followed by the same letter (small letters at $5 \%$ and capital letters at $1 \%$ level of probability) are not significantly different according to Duncan's multiple range test. 
Table 4. Comparison between turfgrass types according to fresh and dry weight of verdure, root, and clipping yield in $100 \mathrm{~cm}^{2}$ area, averaged over 2 years of growing and two sowing times in this study.

\begin{tabular}{|c|c|c|c|c|c|c|c|c|}
\hline \multirow[b]{2}{*}{$\begin{array}{l}\text { Turfgrass } \\
\text { type }^{z}\end{array}$} & \multicolumn{8}{|c|}{ Variable $\left(\mathrm{g} / 100 \mathrm{~cm}^{2}\right)$} \\
\hline & $\begin{array}{c}\text { Total } \\
\text { fresh wt }\end{array}$ & $\begin{array}{c}\text { Total } \\
\text { dry wt }\end{array}$ & $\begin{array}{l}\text { Verdure } \\
\text { fresh wt }\end{array}$ & $\begin{array}{l}\text { Verdure } \\
\text { dry wt }\end{array}$ & $\begin{array}{c}\text { Root } \\
\text { fresh wt }\end{array}$ & $\begin{array}{c}\text { Root } \\
\text { dry wt }\end{array}$ & $\begin{array}{l}\text { Clipping } \\
\text { fresh wt }\end{array}$ & $\begin{array}{c}\text { Clipping } \\
\text { dry wt }\end{array}$ \\
\hline $\mathrm{L}$ & $29.6 b^{y}$ & $9.4 \mathrm{c}$ & $14.3 \mathrm{a}-\mathrm{c}$ & $4.3 \mathrm{a}-\mathrm{e}$ & $15.3 \mathrm{c}$ & $5.1 \mathrm{c}$ & $0.61 \mathrm{bc}$ & $0.18 \mathrm{bc}$ \\
\hline F & $26.5 \mathrm{c}$ & $10.4 \mathrm{bc}$ & $10.5 \mathrm{c}$ & $3.2 \mathrm{e}$ & $16.0 \mathrm{c}$ & $7.2 \mathrm{a}-\mathrm{c}$ & $0.91 \mathrm{a}$ & $0.26 \mathrm{a}$ \\
\hline BAR 11 & $33.4 \mathrm{a}-\mathrm{c}$ & $11.3 \mathrm{a}-\mathrm{c}$ & $14.1 \mathrm{a}-\mathrm{c}$ & $4.2 \mathrm{~b}-\mathrm{e}$ & $19.3 \mathrm{bc}$ & $7.0 \mathrm{a}-\mathrm{c}$ & $0.54 \mathrm{bc}$ & $0.16 \mathrm{bc}$ \\
\hline $\mathrm{MM}$ & $29.6 \mathrm{bc}$ & $10.3 \mathrm{bc}$ & $12.8 \mathrm{c}$ & $3.7 \mathrm{c}-\mathrm{e}$ & $16.8 \mathrm{bc}$ & $6.5 \mathrm{a}-\mathrm{c}$ & $0.77 \mathrm{a}-\mathrm{c}$ & $0.22 \mathrm{a}-\mathrm{c}$ \\
\hline $\mathrm{L}+\mathrm{F}$ & $30.6 \mathrm{bc}$ & $10.2 \mathrm{bc}$ & $14.9 \mathrm{a}-\mathrm{c}$ & $4.7 \mathrm{a}-\mathrm{e}$ & $15.8 \mathrm{c}$ & $5.8 \mathrm{bc}$ & $0.70 \mathrm{a}-\mathrm{c}$ & $0.21 \mathrm{a}-\mathrm{c}$ \\
\hline $\mathrm{P}+\mathrm{C}$ & $38.7 \mathrm{ab}$ & $13.1 \mathrm{a}-\mathrm{c}$ & $19.5 \mathrm{a}$ & $5.7 \mathrm{ab}$ & $19.3 \mathrm{bc}$ & $5.5 \mathrm{a}-\mathrm{c}$ & $0.65 \mathrm{a}-\mathrm{c}$ & $0.19 \mathrm{bc}$ \\
\hline $\mathrm{P}+\mathrm{F}$ & $32.5 \mathrm{bc}$ & $12.6 \mathrm{a}-\mathrm{c}$ & $11.7 \mathrm{c}$ & $3.6 \mathrm{de}$ & $21.3 \mathrm{a}-\mathrm{c}$ & $9.0 \mathrm{ab}$ & $0.80 \mathrm{ab}$ & $0.23 \mathrm{ab}$ \\
\hline $\mathrm{C}+\mathrm{F}$ & $29.8 \mathrm{bc}$ & $10.8 \mathrm{a}-\mathrm{c}$ & $13.7 \mathrm{bc}$ & $4.1 \mathrm{~b}-\mathrm{e}$ & $16.1 \mathrm{c}$ & $7.1 \mathrm{a}-\mathrm{c}$ & $0.61 \mathrm{bc}$ & $0.18 \mathrm{bc}$ \\
\hline $\mathrm{L}+\mathrm{P}+\mathrm{C}+\mathrm{F}$ & $33.0 \mathrm{bc}$ & $11.3 \mathrm{a}-\mathrm{c}$ & $15.0 \mathrm{a}-\mathrm{c}$ & $4.6 \mathrm{a}-\mathrm{e}$ & $18.0 \mathrm{bc}$ & $6.7 \mathrm{a}-\mathrm{c}$ & $0.70 \mathrm{a}-\mathrm{c}$ & $0.20 \mathrm{a}-\mathrm{c}$ \\
\hline
\end{tabular}

${ }^{2} \mathrm{~L}=$ Lolium, $\mathrm{P}=$ Poa, $\mathrm{C}=$ Cynodon, $\mathrm{F}=$ Festuca, $\mathrm{BAR} 11$ and $\mathrm{MM}=$ sport turfgrasses.

'In each column, means followed by the same letters are not significantly different according to Duncan's multiple range test at $1 \%$ level.

Table 5. Comparison between turfgrass types according to tiller density, rooting depth, and chlorophyll index in $100 \mathrm{~cm}^{2}$ area, averaged over 2 years of growing and two sowing seasons in this study.

\begin{tabular}{lccc}
\hline \multirow{2}{*}{$\begin{array}{l}\text { Turfgrass } \\
\text { type }\end{array}$} & \multicolumn{3}{c}{ Variable } \\
\cline { 2 - 4 } & $\begin{array}{c}\text { Tiller density } \\
\left(\text { no./100 cm }{ }^{2}\right.\end{array}$ & $\begin{array}{c}\text { Mean rooting } \\
\text { depth }(\mathrm{cm})\end{array}$ & $\begin{array}{c}\text { Chlorophyll index after } \\
\text { summer }\left(\mathrm{mg} / 100 \mathrm{~cm}^{2}\right)\end{array}$ \\
\hline $\mathrm{L}$ & $393.8 \mathrm{~b}^{\mathrm{y}}$ & $6.8 \mathrm{a}-\mathrm{c}$ & $23.9 \mathrm{ab}$ \\
$\mathrm{P}$ & $577.3 \mathrm{ab}$ & $5.4 \mathrm{c}$ & $29.8 \mathrm{ab}$ \\
$\mathrm{C}$ & $585.6 \mathrm{ab}$ & $5.4 \mathrm{c}$ & $29.7 \mathrm{ab}$ \\
$\mathrm{F}$ & $740.4 \mathrm{a}$ & $5.6 \mathrm{bc}$ & $18.2 \mathrm{~b}$ \\
BAR 11 & $352.7 \mathrm{~b}$ & $6.8 \mathrm{a}-\mathrm{c}$ & $26.8 \mathrm{ab}$ \\
MM & $420.9 \mathrm{~b}$ & $7.3 \mathrm{ab}$ & $26.3 \mathrm{ab}$ \\
$\mathrm{L}+\mathrm{P}$ & $332.0 \mathrm{~b}$ & $7.0 \mathrm{a}-\mathrm{c}$ & $22.2 \mathrm{ab}$ \\
$\mathrm{L}+\mathrm{C}$ & $298.9 \mathrm{~b}$ & $7.5 \mathrm{a}$ & $27.6 \mathrm{ab}$ \\
$\mathrm{L}+\mathrm{F}$ & $345.7 \mathrm{~b}$ & $6.9 \mathrm{a}-\mathrm{c}$ & $24.8 \mathrm{ab}$ \\
$\mathrm{P}+\mathrm{C}$ & $507.1 \mathrm{ab}$ & $6.4 \mathrm{a}-\mathrm{c}$ & $33.2 \mathrm{a}$ \\
$\mathrm{P}+\mathrm{F}$ & $598.7 \mathrm{ab}$ & $5.5 \mathrm{bc}$ & $19.7 \mathrm{~b}$ \\
$\mathrm{C}+\mathrm{F}$ & $447.4 \mathrm{~b}$ & $6.6 \mathrm{a}-\mathrm{c}$ & $22.8 \mathrm{ab}$ \\
$\mathrm{L}+\mathrm{P}+\mathrm{C}+\mathrm{F}$ & $387.0 \mathrm{~b}$ & $8.1 \mathrm{a}$ & $23.1 \mathrm{ab}$ \\
\hline
\end{tabular}

${ }^{2} \mathrm{~L}=$ Lolium, $\mathrm{P}=$ Poa, $\mathrm{C}=$ Cynodon, $\mathrm{F}=$ Festuca, $\mathrm{BAR} 11$ and $\mathrm{MM}=$ sport turfgrasses.

'In each column, means followed by the same letters are not significantly different according to Duncan's multiple range test at $1 \%$ level.
It can be concluded that superiority of fall sowing may be due to higher root growth and clipping yield (new shoot growth). Furthermore, the tolerance to environmental stresses which induced by after sowing cold weather (Pollock et al., 1993) resulted in higher chlorophyll content after winter compared to spring sowing. Even C, which is a warmseason turfgrass, was greener after winter in fall sowing compared to spring sowing. This may be also due to tolerance inducing effect of cold weather in early stages of development (Pollock et al., 1993). This is in contrast to previous reports where there was a preference for sowing warm-season turfgrasses in spring due to high temperature requirements for seed germination (Beard, 1973). Hensler et al. (1999) with $C$ cultivars, indicated that morphological immaturity in late sowing might reduce cold tolerance. These different results may be due

Table 6. Interaction of sowing times and turfgrass types effects on fresh and dry weight of verdure, root, and clipping yield in $100 \mathrm{~cm}^{2}$ area averaged over 2 years of growing in this study.

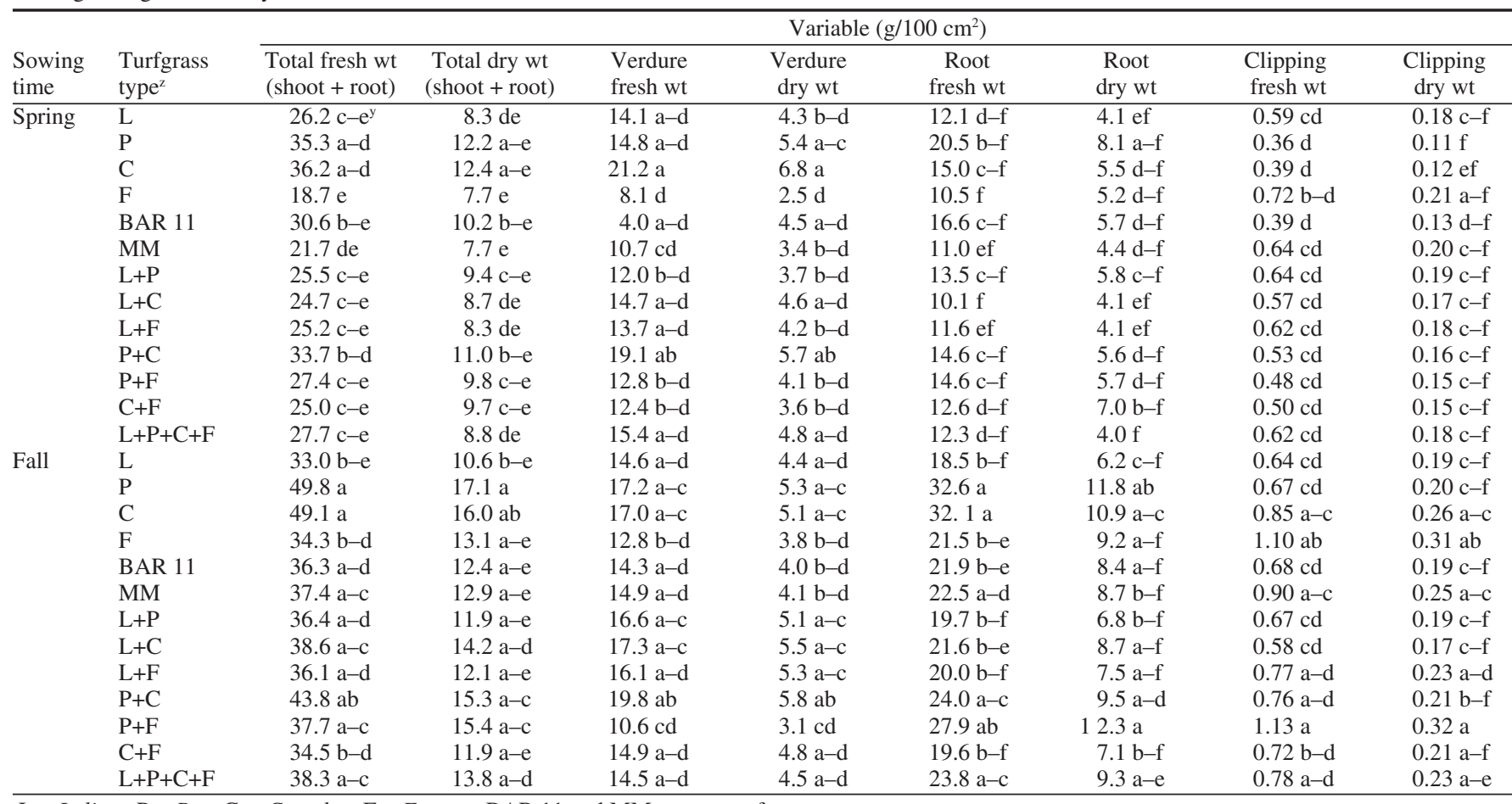

${ }^{2} \mathrm{~L}=$ Lolium, $\mathrm{P}=$ Poa, $\mathrm{C}=$ Cynodon, $\mathrm{F}=$ Festuca, $\mathrm{BAR} 11$ and $\mathrm{MM}=$ sport turfgrasses.

'In each column, means followed by the same letters are not significantly different according to Duncan's multiple range test at $1 \%$ level. 
Table 7. Interaction of sowing times and turfgrass types effects on tiller density, rooting depth, and chlorophyll index in $100 \mathrm{~cm}^{2}$ area, averaged over 2 years of growing in this study.

\begin{tabular}{|c|c|c|c|c|c|}
\hline \multirow[b]{2}{*}{$\begin{array}{l}\text { Sowing } \\
\text { times }\end{array}$} & \multirow[b]{2}{*}{$\begin{array}{l}\text { Turfgrass } \\
\text { type }^{z}\end{array}$} & \multicolumn{4}{|c|}{ Variable } \\
\hline & & $\begin{array}{l}\text { Tiller density } \\
\left(\text { no. } / 100 \mathrm{~cm}^{2}\right)\end{array}$ & $\begin{array}{c}\text { Mean rooting } \\
\text { depth }(\mathrm{cm})\end{array}$ & $\begin{array}{l}\text { Chlorophyll index: } \\
\text { after summer } \\
\left(\mathrm{mg} / 100 \mathrm{~cm}^{2}\right)\end{array}$ & $\begin{array}{l}\text { Chlorophyll index: } \\
\text { after winter } \\
\left(\mathrm{mg} / 100 \mathrm{~cm}^{2}\right)\end{array}$ \\
\hline \multirow[t]{13}{*}{ Spring } & $\mathrm{L}$ & $317.8 b^{y}$ & $6.2 \mathrm{~b}-\mathrm{d}$ & $23.8 \mathrm{a}-\mathrm{d}$ & $4.3 \mathrm{bc}$ \\
\hline & $\mathrm{P}$ & $563.4 \mathrm{ab}$ & $4.8 \mathrm{~d}$ & $21.7 \mathrm{a}-\mathrm{d}$ & $6.1 \mathrm{ab}$ \\
\hline & $\mathrm{C}$ & $638.5 \mathrm{ab}$ & $5.8 \mathrm{~b}-\mathrm{d}$ & $36.9 \mathrm{ab}$ & $5.6 \mathrm{a}-\mathrm{c}$ \\
\hline & $\mathrm{F}$ & $797.9 \mathrm{a}$ & $5.4 \mathrm{~cd}$ & $8.8 \mathrm{~d}$ & $2.9 \mathrm{c}$ \\
\hline & BAR 11 & $303.2 \mathrm{~b}$ & $6.6 b-d$ & $25.1 \mathrm{a}-\mathrm{d}$ & $5.9 \mathrm{a}-\mathrm{c}$ \\
\hline & MM & $379.4 \mathrm{ab}$ & $6.7 \mathrm{~b}-\mathrm{d}$ & $18.6 \mathrm{~cd}$ & $4.0 \mathrm{bc}$ \\
\hline & $\mathrm{L}+\mathrm{P}$ & $325.9 \mathrm{~b}$ & $6.7 b-d$ & $21.6 \mathrm{a}-\mathrm{d}$ & $5.2 \mathrm{a}-\mathrm{c}$ \\
\hline & $\mathrm{L}+\mathrm{C}$ & 294.8 b & $6.7 b-d$ & $24.3 \mathrm{a}-\mathrm{d}$ & $5.3 \mathrm{a}-\mathrm{c}$ \\
\hline & $\mathrm{L}+\mathrm{F}$ & $299.3 \mathrm{~b}$ & $6.6 \mathrm{~b}-\mathrm{d}$ & $27.8 \mathrm{a}-\mathrm{c}$ & $5.0 \mathrm{a}-\mathrm{c}$ \\
\hline & $\mathrm{P}+\mathrm{C}$ & $550.0 \mathrm{ab}$ & $6.4 b-d$ & $27.2 \mathrm{a}-\mathrm{c}$ & $5.2 \mathrm{a}-\mathrm{c}$ \\
\hline & $\mathrm{P}+\mathrm{F}$ & $734.0 \mathrm{ab}$ & $4.8 \mathrm{~d}$ & $17.7 \mathrm{~cd}$ & $6.4 \mathrm{a}-\mathrm{c}$ \\
\hline & $\mathrm{C}+\mathrm{F}$ & $468.4 \mathrm{ab}$ & $4.9 \mathrm{~d}$ & $21.1 \mathrm{a}-\mathrm{d}$ & $4.5 \mathrm{bc}$ \\
\hline & $\mathrm{L}+\mathrm{P}+\mathrm{C}+\mathrm{F}$ & $347.4 \mathrm{~b}$ & $6.3 \mathrm{~b}-\mathrm{d}$ & $19.9 b-d$ & $5.4 \mathrm{a}-\mathrm{c}$ \\
\hline \multirow[t]{13}{*}{ Fall } & $\mathrm{L}$ & $469.9 \mathrm{ab}$ & $7.4 \mathrm{a}-\mathrm{d}$ & $24.1 \mathrm{a}-\mathrm{d}$ & $7.9 \mathrm{a}-\mathrm{c}$ \\
\hline & $\mathrm{P}$ & $591.1 \mathrm{ab}$ & $6.1 \mathrm{~b}-\mathrm{d}$ & $38.0 \mathrm{ab}$ & $10.3 \mathrm{ab}$ \\
\hline & $\mathrm{C}$ & $532.7 \mathrm{ab}$ & $5.0 \mathrm{~d}$ & $22.5 \mathrm{a}-\mathrm{d}$ & $10.2 \mathrm{a}-\mathrm{c}$ \\
\hline & $\mathrm{F}$ & $682.9 \mathrm{ab}$ & $5.8 \mathrm{~b}-\mathrm{d}$ & $27.6 \mathrm{a}-\mathrm{c}$ & $5.3 \mathrm{a}-\mathrm{c}$ \\
\hline & BAR 11 & $402.2 \mathrm{ab}$ & $7.1 \mathrm{~b}-\mathrm{d}$ & $28.5 \mathrm{a}-\mathrm{c}$ & $8.9 \mathrm{a}-\mathrm{c}$ \\
\hline & MM & $462.4 \mathrm{ab}$ & $7.9 \mathrm{a}-\mathrm{c}$ & $34.0 \mathrm{a}-\mathrm{c}$ & $6.1 \mathrm{a}-\mathrm{c}$ \\
\hline & $\mathrm{L}+\mathrm{P}$ & $338.2 \mathrm{~b}$ & $7.3 \mathrm{~b}-\mathrm{d}$ & $22.8 \mathrm{a}-\mathrm{d}$ & $11.7 \mathrm{a}$ \\
\hline & $\mathrm{L}+\mathrm{C}$ & $303.0 \mathrm{~b}$ & $8.3 \mathrm{ab}$ & $31.0 \mathrm{a}-\mathrm{c}$ & $11.8 \mathrm{a}$ \\
\hline & $\mathrm{L}+\mathrm{F}$ & $392.1 \mathrm{ab}$ & $7.2 \mathrm{~b}-\mathrm{d}$ & $21.8 \mathrm{a}-\mathrm{d}$ & $4.9 \mathrm{a}-\mathrm{c}$ \\
\hline & $\mathrm{P}+\mathrm{C}$ & $464.3 \mathrm{ab}$ & $6.3 \mathrm{~b}-\mathrm{d}$ & $39.2 \mathrm{a}$ & $10.3 \mathrm{ab}$ \\
\hline & $\mathrm{P}+\mathrm{F}$ & $463.4 \mathrm{ab}$ & $6.2 \mathrm{~b}-\mathrm{d}$ & $21.7 \mathrm{a}-\mathrm{d}$ & $5.6 \mathrm{a}-\mathrm{c}$ \\
\hline & $\mathrm{C}+\mathrm{F}$ & $426.3 \mathrm{ab}$ & $8.3 \mathrm{ab}$ & $24.6 \mathrm{a}-\mathrm{d}$ & $6.2 \mathrm{a}-\mathrm{c}$ \\
\hline & $\mathrm{L}+\mathrm{P}+\mathrm{C}+\mathrm{F}$ & $426.5 \mathrm{ab}$ & $9.8 \mathrm{a}$ & $26.3 \mathrm{a}-\mathrm{d}$ & $4.5 \mathrm{bc}$ \\
\hline
\end{tabular}

${ }^{2} \mathrm{~L}=$ Lolium, $\mathrm{P}=$ Poa, $\mathrm{C}=$ Cynodon, $\mathrm{F}=$ Festuca, $\mathrm{BAR} 11$ and $\mathrm{MM}=$ sport turfgrasses.

'In each column, means followed by the same letters are not significantly different according to Duncan's multiple range test at $1 \%$ level.

to genotype and geographical location differences. Further experiments are needed to better understand of this phenomenon.

In our study, L had not good characteristics, especially its tiller density was low, and $\mathrm{P}$ and $\mathrm{C}$ were slow growing. Lowering the growth may be applicable in reducing the mowing frequency. The high tiller density observed in $\mathrm{F}$ may be due to compact growth of this turfgrass (Beard, 1973). Festuca had a good growth similar to other rapid growing turfgrass types, because of adaptation to cold weather in fall sowing (Pollock et al., 1993). This turfgrass was very sensitive to high temperatures and low humidity. Festuca had the lowest chlorophyll index after winter in spring culture. It may be due to previous heat injury to this turfgrass during summer and reducing the cold tolerance during winter. Sport turfgrasses were not suitable in this study, because of this fact that sport turfs are produce for special uses (especially in the site of production). These sport turfgrasses are consisted of some cultivars (see materials and methods) that may not be suitable for studied area, or may have inappropriate mixing percentages.

Among seed mixtures, $\mathrm{L}+\mathrm{P}, \mathrm{L}+\mathrm{C}, \mathrm{L}+\mathrm{F}$, and $\mathrm{L}+\mathrm{P}+\mathrm{C}+\mathrm{F}$ were good to excellent turfgrasses in our study but had low tiller density. Cynodon $+\mathrm{F}$ and $\mathrm{P}+\mathrm{F}$ did not show good characteristics in studied area. Poa $+\mathrm{F}$ was a weak turfgrass and similar to $\mathrm{F}$ it was not tolerant to warm temperatures during summer. It is not in accordance with Misiha (1991) results with Poa 'Entopper' and Festuca 'Hareld' seed mixture. These contradictory results may be due to differences in genotypes, geographical location, and environmental conditions. Poa $+\mathrm{C}$ was an excellent turfgrass mixture in this study. This type had a very good tolerance to both high and low temperatures. This is in accordance with Dunn et al. (1994) result.

\section{Conclusions}

Poa, $\mathrm{C}$, and $\mathrm{P}+\mathrm{C}$ in addition to having several good characteristics, have a high tiller density. High tiller density will result in good wear tolerance (Lush, 1990). Poa + Chad been graded best according to visual quality (Table 2 ). Thus, fall sowing of $\mathrm{P}+\mathrm{C}$ is recommended in the areas with soil and environmental conditions similar to our research site. This indicates the possibility of using cool-warm-season seed mixtures alternatively in overseeding programs.

\section{Literature Cited}

Beard, J.B. 1973. Turfgrass: Science and culture. Prentice-Hall, Inc., Englewood Cliffs, N.J.

Cooper, R.J. and L.A. Spokas. 1991. Growth, quality, and foliar iron concentration of kentucky bluegrass treated with chelated iron sources. J. Amer. Soc. Hort. Sci. 116:798-801.

Dunn, J.H., D.D. Minner, B.F. Fresenburg, and S.S. Bughrara. 1994. Bermudagrass and cool season turfgrass mixtures: Response to simulated traffic. Agron. J. 86:10-16.

Garling, D.C. and M.J. Boehm. 2001. Temporal effects of compost and fertilizer applications on nitrogen fertility of golf course turfgrass. Agron. J. 93:548-555.

Gooding, M.J. and A.J. Newell. 1991. Analysis of temporal changes in cultivars of Poa pratensis subjected to football-type wear. J. Sports Turf Res. Inst. 67:113-119.

Hensler, K.L., M.D. Richardson, and J.R. Bailey. 1999. Implications of seeded bermudagrass planting date and morphology on cold tolerance. Res. Ser. Arkan. Agr. Expt. Sta. 466:69-71.

Horst, G.L., M.C. Engelke, and W. Meyers. 1984. Assessment of visual evaluation techniques. Agron. J. 76:619-622.

Horwitz, W. 1975. Official methods of analysis. $12^{\text {th }}$ ed. AOAC, Wash., D.C.

Lush, W.M. 1990. Turf growth and performance evaluation based on turf biomass and tiller density. Agron. J. 82:505-511.

Mazur, A.R. 1988. Influence of plant growth regulators on transition of bermudagrass putting green overseeded with perennial ryegrass. J. Amer. Soc. Hort. Sci. 113:367-373.

Misiha, A. 1991. Effect of cool season turfgrasses seed mixtures on lawn characteristics. Bul. Facul. Agr. Univ. Cairo 42:401-414.

Newell, A.J., F.E.M. Crossley, and A.C. Jones. 1996. Selection of grass species, cultivars and mixtures for lawn tennis courts. J. Sports Turf Res. Inst. 72:42-60.

Pollock, C.J., C.F. Eagles, C.J. Howarth, P.H.D. Schünmann, and J.L. Stoddart. 1993. Temperature stress, p. 109-132. In: L. Fowden, T. Mansfield, and J. Stoddart (eds.). Plant adaptation to environmental stress. Chapman and Hall Inc., London.

Skirde, W. 1989. Performance of new cultivars of tall fescue (Festuca arundinacea) in pure sowings and mixtures, p. 166. In: 1992 Horticultural abstracts. CAB Intl., Wallingford, Oxford, U.K. 